\title{
C-Reactive Protein Concentration in Canine Idiopathic Polyarthritis
}

\author{
Koichi OHNO ${ }^{1)}$, Yukiko YOKOYAMA ${ }^{1)}$, Ko NAKASHIMA ${ }^{1)}$, Asuka SETOGUCHI ${ }^{1)}$, Yasuhito FUJINO $^{1)}$ and \\ Hajime TSUJIMOTO ${ }^{1)}$ \\ ${ }^{1)}$ Department of Veterinary Internal Medicine, Graduate School of Agricultural and Life Sciences, The University of Tokyo, 1-1-1 Yayoi, \\ Bunkyo-ku, Tokyo 113-8657, Japan
}

(Received 10 April 2006/Accepted 18 August 2006)

ABSTRACT. To investigate the clinical utility of C-reactive protein (CRP) in idiopathic polyarthritis (IPA), its concentration was measured in dogs with IPA. The CRP concentration was markedly increased in all the IPA dogs at the time of diagnosis and decreased significantly in response to the initial corticosteroid treatment; this indicated that CRP can be used as an index for therapeutic response in IPA cases. Furthermore, at 6 months after the diagnosis, a significant association was observed between the CRP concentration at follow-up (6-13 days after the treatment was started) and the frequency of medication ("no or seldom-medicated (NSM)" groups or "continuing medication (CM)" groups). These results suggest that the initial response of CRP to corticosteroid treatment may be a prognostic factor of canine IPA.

KEY WORDS: canine, C-reactive protein, idiopathic polyarthritis.

The etiology of canine inflammatory joint diseases is either infectious or noninfectious. Noninfectious arthritis includes immune-mediated arthritis, which can be subdivided as erosive and nonerosive arthritis according to the radiographic and histopathologic appearance of the joints [14]. Rheumatoid arthritis (RA) is the most important erosive and noninfectious polyarthritis [1]. Nonerosive and noninfectious arthritis includes idiopathic polyarthritis (IPA) [4], systemic lupus erythematosus (SLE) [2], and polyarthritis/polymyositis syndrome [3]. Among these, IPA is the most common type of immune-mediated joint disease. In contrast, RA is the most common, important arthritis in human and has been investigated for its pathogenesis.

Canine IPA was first reported by Bennett in the mid 1970 s as a disease of canine joints without any obvious etiology [4]. The clinical features of IPA include persistent and episodic fever, anorexia, and malaise accompanied by lameness/inability to walk of varying severity $[4,11,15]$. IPA is diagnosed when radiographic abnormalities are absent and the synovial fluid examination is indicative of inflammation, and after the exclusion of other types of inflammatory joint diseases. Due to the lack of specific laboratory tests and its vague symptoms, IPA is sometimes difficult to diagnose and is known to be the most common cause of fever of undetermined origin [8].

The treatment of canine IPA usually comprises corticosteroids at immunosuppressive doses and other immunosuppressive drugs for refractory cases [4, 11, 15]. Most IPA dogs respond to steroid treatment and symptomatic control may be achieved, although relapses occur. Bennett reported that the prognosis of IPA in dogs is generally better than that of other types of inflammatory joint disease such as RA and SLE [4]. However, not much is known about the long-term outcomes and the prognostic factors of canine IPA.

C-reactive protein (CRP) is one of the important acute phase proteins, and its serum concentration increases rap- idly in response to inflammation or tissue destruction [5]. CRP is currently the gold standard inflammatory marker for estimation of the severity of diseases in human medicine. CRP has also been used as a predictor of survival in some neoplastic conditions [17] and of risk for myocardial infarction and stroke $[6,20]$. Recently, the CRP concentration was measured in dogs and it was shown to be elevated in several conditions such as surgical trauma [13], inflammatory bowel diseases (IBD) [12], pancreatitis [10], and autoimmune hemolytic anemia (AIHA) [18]. In Japan, a nepherometric immunoassay that is specific for canine CRP was developed in 1998, and the data of canine plasma CRP concentrations were accumulated for more than 10 years [13]. The aim of this study is to determine the CRP concentration in IPA dogs through a retrospective study in order to evaluate its clinical utility.

\section{MATERIALS AND METHODS}

Cases: We reviewed the medical records of 38 dogs that were diagnosed with IPA at the Veterinary Medical Center of the University of Tokyo (VMC-UT) between April 2002 and March 2005. The diagnostic criteria for the inclusion of cases in the study were nonerosive polyarthritis that was confirmed by a radiographic assessment of the joints, noninfectious polyarthritis that was confirmed by cytologic analysis and/or a bacterial culture of synovial fluids (SF), which were obtained by multiple arthrocentesis, and the absence of RA, SLE, polyarthritis/polymyositis syndrome, and polyarthritis-meningitis syndrome. SF was obtained by arthrocentesis of at least 3 joints, usually the carpus, the hock, and the stifle joints, and was performed on most patients without premedication or induction of anesthesia. The direct cytologic examination of Wright-Giemsa-stained smears of SF was performed. In addition, RA was considered to be absent if no erosive changes were identified on radiographs of the 
joints. SLE was excluded when there was no evidence of the involvement of other body systems based on clinical or laboratory examinations and when the test for serum antinuclear antibody was negative. Anti-nuclear antibody was detected using a commercially available kit (Fluoro Hepana test, MBL, Aichi, Japan), and a titer of more than 1:80 was considered positive.

Each dog was treated with an immunosuppressive dose of prednisolone $(2-4 \mathrm{mg} / \mathrm{kg} /$ day) as the initial treatment for at least 14 days. Subsequently, the dose of prednisolone was gradually reduced by $50 \%$ at 2 - to 4 -week intervals, and finally its administration was stopped in dogs in whom no relapse was observed. However, when the disease relapsed, another course of prednisolone treatment with or without other immunosuppressive drugs was administered. The concomitant immunosuppressive agents used were $2 \mathrm{mg} / \mathrm{kg} /$ day of azathioprine, $2.5-5 \mathrm{mg} / \mathrm{kg}$ /day leflunomide, and 20 $40 \mathrm{mg} / \mathrm{kg} /$ day mycophenolate mofetil.

Retrospective study procedures: Details obtained from the medical records of the dogs included their age, breed, sex with neuter status, chief complaint, complications, if any, and body temperature on the day of diagnosis. In addition, we obtained the WBC counts in the peripheral blood and the plasma CRP concentrations on the day of diagnosis and follow-up (between 6-13 days after the treatment was started), wherever possible. The plasma CRP concentration was measured using a canine CRP measurement kit (Laser CRP-2, Arrows Co., Ltd., Osaka, Japan) according to the manufacturer's instruction. The reference range of the plasma CRP concentration was considered as $0-1 \mathrm{mg} / \mathrm{d} l$ according to the manufacturer's data sheet and a previous report [13]. The reference range of WBC counts in our laboratory was $6,000-17,000 / \mu l$.

The frequency of medication was evaluated in each dog at 6 months after the treatment was started. It was described as either "no or seldom-medicated (NSM)" or "continuing medication (CM)". Dogs who were not receiving any medication or those who were admistered prednisolone or other immunosuppressive drugs occasionally (at a maximum interval of 14 days) to control the clinical signs of IPA were included in the NSM group. Dogs who were administered medications to control the clinical signs more frequently, that is, medications were administered even earlier than every 14 days were grouped in the CM group.

Statistical analysis: Differences in the WBC counts and plasma CRP concentrations at the time of diagnosis and that at follow-up were examined by paired $t$ test. A CRP concentration greater than the measurement limit $(>20 \mathrm{mg} / \mathrm{d} l)$ was considered as $20 \mathrm{mg} / \mathrm{d} l$ for statistical analysis. Continuous data were compared by Student's $t$ test in the case of equal variance. The Welch $t$ test was used to compare continuous data with unequal variance. Frequency incidences of the two groups (NSM and CM) were analyzed by Fisher's exact test. The associations between CRP and other variables were examined using Pearson's product-moment correlation coefficient. A P value of less than 0.05 was considered statistically significant.

\section{RESULTS}

During the study period, 38 dogs fulfilled the diagnostic criteria of IPA. Between April 2002 and March 2005, a total of 3,477 dogs were referred to the VMC-UT; this translates into an IPA incidence of $1.09 \%$ in our hospital. The median age of the dogs was 5.5 years (mean, 6.0 years; range, 1-13 years). Of the $38 \mathrm{dogs}, 12$ were male (10 entire and 2 neutered) and 26 were female (14 entire and 12 neutered). Thirty-five dogs that represented 13 pure breeds and 3 dogs that represented mixed breeds were affected. The most commonly affected breeds were Miniature Dachshund $(n=11)$, Welsh Corgi $(n=5)$, and Shih Tzu $(n=5)$. The median body weight was $4.7 \mathrm{~kg}$ (mean weight, $6.1 \mathrm{~kg}$; range, $2.0-14.5 \mathrm{~kg})$.

Several clinical symptoms related to IPA were noted, including pyrexia in 27 dogs $(71 \%)$, lameness/inability to walk in $18 \mathrm{dogs}(47 \%)$, and inappetance/signs of depression in 15 dogs (39\%); these were the most common chief signs. Five dogs (25\%) exhibited joint pain and 11 dogs were hospitalized for the initial 3 to 6 days due to their serious condition. All 38 IPA dogs, including the hospitalized dogs, responded to the initial prednisolone treatment within 14 days and their clinical signs improved.

The CRP concentration was elevated $(>1 \mathrm{mg} / \mathrm{d} l)$ in all the IPA dogs, and the mean CRP concentration at the time of diagnosis was $13.9 \pm 6.1 \mathrm{mg} / \mathrm{d} l$. The CRP concentrations of the 11 dogs $(29 \%)$ were greater than the maximum measurement limit ( $>20 \mathrm{mg} / \mathrm{d} l$; Fig. 1A). On the other hand, the
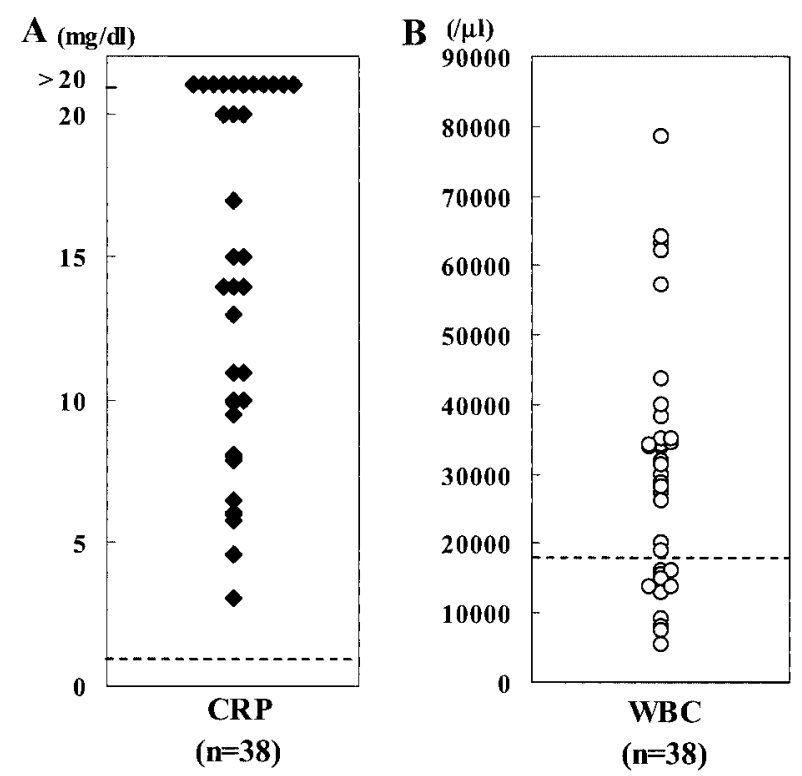

Fig. 1. Plasma CRP concentrations (A) and WBC counts (B) at the time of diagnosis in IPA dogs. The dashed line represents the maximum limit of the reference ranges of CRP $(1 \mathrm{mg} / \mathrm{d} l)$ and WBC $(17,000 / \mu l)$. CRP concentration was elevated in all the IPA dogs, and it was greater than the maximum measurement limit $(>20 \mathrm{mg} / \mathrm{d} l)$ in $11 \operatorname{dogs}(\mathrm{A})$. The mean WBC count of the IPA dogs was $29,897 \pm 17,576 / \mu l$ and 26 dogs showed leukocytosis (B). 


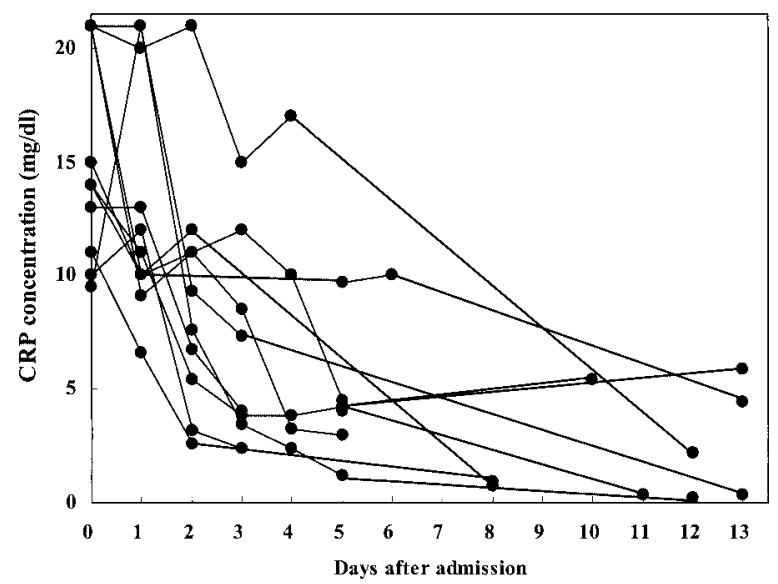

Fig. 2. Decrease in the plasma CRP concentration in 11 hospitalized IPA dogs within 13 days after the treatment was started. The CRP concentration decreased to $<5 \mathrm{mg} / \mathrm{d} l$ in 9 of the 11 dogs within 5 days and then returned to the reference range in 5 of the 10 dogs within 6-13 days after admission.

mean WBC counts of the IPA dogs $(n=38)$ was $29,897 \pm$ $17,576 / \mu l$, and only $26 \operatorname{dogs}(68 \%)$ showed leukocytosis (Fig. 1B). The CRP concentrations were serially monitored in the 11 hospitalized dogs. This concentration dramatically decreased to $<5 \mathrm{mg} / \mathrm{d} l$ in 9 of the $11 \mathrm{dogs}(82 \%)$ within 5 days after the steroid treatment was started. It then returned to the reference range $(<1 \mathrm{mg} / \mathrm{d} l)$ in 5 of the 10 dogs $(50 \%)$ within 6-13 days after their admission (Fig. 2). When the CRP concentration in the IPA dogs $(n=35)$ at the time of diagnosis was compared with that at follow-up (Fig. 3), a significant $(\mathrm{P}<0.05)$ decrease was observed at follow-up $(12$ \pm 3 days after the treatment was started; range, 7-14 days), and the concentration returned to the reference range in 17 dogs (49\%). In contrast, the mean WBC count at follow-up $(\mathrm{n}=33)$ was $31,470 \pm 19,135 / \mu l$, and compared to the count at the time of diagnosis, no significant decrease was observed. The WBC counts of 25 of the 33 dogs (76\%) were more than the reference range even at follow-up. No correlation was observed between the CRP concentration and the WBC counts at the time of diagnosis $(\mathrm{r}=0.197)$. However, a significant correlation was observed between these parameters at follow-up $(\mathrm{r}=0.603, P<0.001)$. No association was observed between the CRP concentration and the body temperature of the IPA dogs (mean, $39.6 \pm 0.7^{\circ} \mathrm{C}$; range, $38.4-40.9^{\circ} \mathrm{C}$ ) or any other laboratory findings (data not shown).

At 6 months after the treatment was started, 10 of 26 dogs $(38.5 \%)$ were included in the NSM group and 16 dogs (61.5\%) were included in the CM group. The mean CRP concentration of the dogs in the NSM group at follow-up (13 \pm 2 days after the treatment was started) was $0.26 \pm 0.23 \mathrm{mg} /$ $\mathrm{d} l$; this value was significantly $(P<0.01)$ lower than that of the dogs in the CM group $(1.86 \pm 1.69 \mathrm{mg} / \mathrm{d} l$ at $11 \pm 3$ days after the treatment was started; Fig. 4). In contrast, no significant difference was observed in the WBC count between
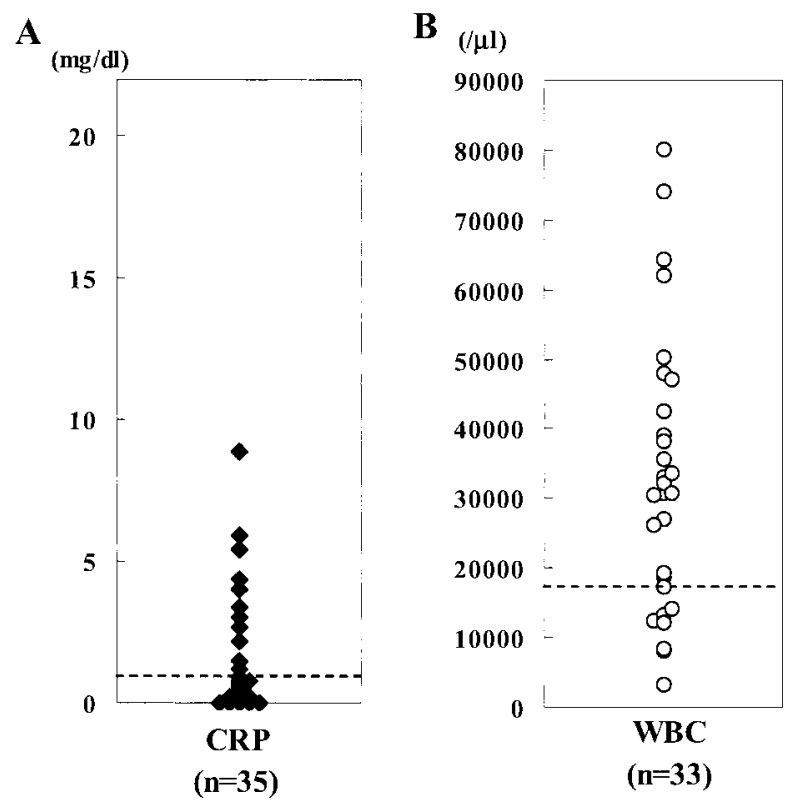

Fig. 3. Plasma CRP concentrations (A) and WBC counts (B) at the initial follow-up in the IPA dogs. The dashed line represents the maximum limit of the reference ranges of CRP $(1 \mathrm{mg} / \mathrm{d} l)$ and $\mathrm{WBC}(17,000 / \mu l)$. The CRP concentration returned to the reference range in 17 dogs $(49 \%)$ at follow-up $(12 \pm 3$ days after the treatment was started; range, 7-14 days). The WBC counts of 25 of the 33 dogs $(76 \%)$ persisted above the reference range; the mean WBC count at follow-up was $31,470 \pm 19,135 / \mu l$.

the NSM group $(22,040 \pm 11,429 / \mu l)$ and the CM group $(32,953 \pm 18,267 / \mu l$; Fig. 4). Furthermore, the IPA dogs with an elevated CRP concentration $(\geq 1 \mathrm{mg} / \mathrm{d} l)$ at follow-up showed poor disease prognosis and required more frequent $(P<0.05)$ medication than the dogs with a normal $(<1 \mathrm{mg} / \mathrm{d} l)$ CRP concentration, as determined by Fisher's exact test (Table 1). No correlation was observed between leukocytosis and the medication status at 6 months after the treatment was started $(P=0.237)$. Radiographs of the joints at 6 months after the diagnosis were assessed in 15 dogs ( 8 from the NSM and 7 from the CM groups); however, no erosive changes were detected.

\section{DISCUSSION}

The aim of our study was to determine the plasma CRP concentration in the IPA dogs and to evaluate its clinical utility for the treatment response, and prognosis in IPA cases. We observed increased CRP concentrations in all the IPA dogs included in this study. This finding was expected based on previous studies that reported elevated CRP concentrations in dogs with other inflammatory diseases $[10$, $12,13,18]$. However, all the IPA dogs showed remarkable increase in the CRP levels; these levels in 11 dogs (29\%) were greater than the measurement limit. This indicated that CRP could be used as a diagnostic aid for canine IPA. Furthermore, the CRP levels decreased rapidly in response to 
A

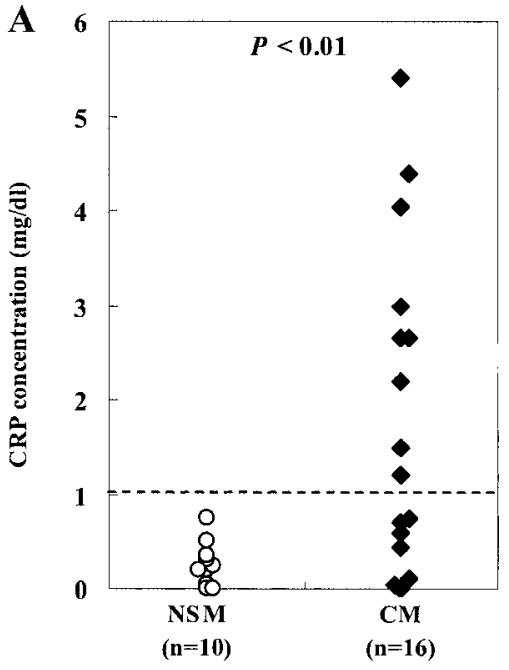

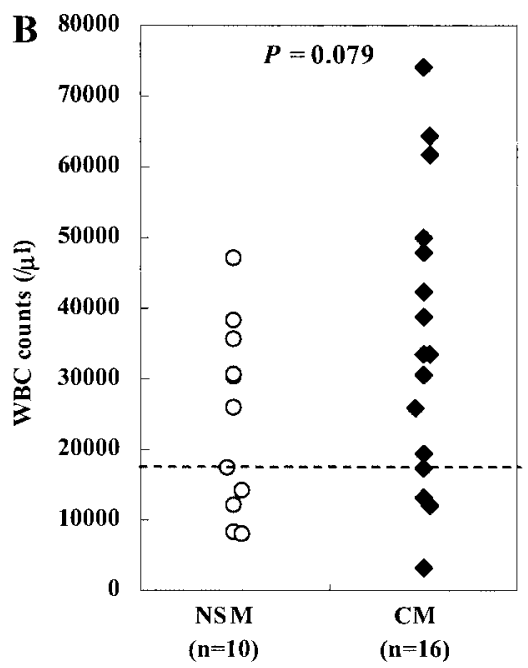

Fig. 4. CRP concentrations and WBC counts at the initial follow-up in the IPA dogs in the "no or seldom-medicated (NSM)" and "continuing medication (CM)" groups; the dogs were classified into the respective groups at 6 months after the diagnosis. The dashed line represents the maximum limit of the reference ranges of CRP $(1 \mathrm{mg} / \mathrm{d} l)$ and WBC $(17,000 / \mu l)$. The mean CRP concentration in the NSM group was $0.26 \pm 0.23 \mathrm{mg} / \mathrm{d} l$; this value was significantly $(P<0.01)$ lower than that in the CM group $(1.86 \pm 1.69 \mathrm{mg} / \mathrm{d} l)$. The mean WBC count in the NSM group was $22,040 \pm 11,429 / \mu l$, and no significant difference was observed between the NSM group and the CM group (mean WBC count, 32,953 $\pm 18,267 / \mu l$ ).

Table 1. CRP concentrations at follow-up and medication status at 6 months after the diagnosis of IPA in dogs

\begin{tabular}{ccccc}
\hline & & \multicolumn{3}{c}{ No. of patients } \\
\cline { 3 - 5 } & & $\mathrm{NSM}^{\mathrm{b})}$ & $\mathrm{CM}^{\mathrm{b})}$ & $P$ value $^{\mathrm{c})}$ \\
\hline CRP & $<1 \mathrm{mg} / \mathrm{d} l$ & 10 & 7 & \\
Concentration $^{\text {a) }}$ & $\geq 1 \mathrm{mg} / \mathrm{d} l$ & 0 & 9 & $<0.01$ \\
\hline WBC & $<17,000 / \mu l$ & 4 & 3 & \\
Counts $^{\text {a) }}$ & $\geq 17,000 / \mu l$ & 6 & 13 & 0.237 \\
\hline
\end{tabular}

a) Determined at initial follow-up (6-13 days after the treatment was started).

b) NSM; no or seldom-medicated group, CM; continuing medication group.

c) Determined by Fisher's exact test.

steroid treatment, whereas the WBC counts did not; this indicated that CRP could be a useful index for evaluating treatment responses.

In our study, the most commonly observed chief sign in 38 IPA dogs was pyrexia (71\%), while lameness/inability to walk $(47 \%)$ and joint pain $(25 \%)$ were less frequently observed. These results differ from those of previous reports in which lameness/inability to walk was the most commonly observed clinical sign $[4,15]$. The discrepancy may be partly explained by the fact that small- and mediumbreed dogs were overrepresented in our study, whereas large-breed dogs were predominant in previous reports. Sometimes, differentiating IPA from other diseases that result in lameness is difficult because of the vague clinical signs of IPA. Therefore, CRP measurement may be used effectively to facilitate the differential diagnosis between immune-mediated polyarthritis, including IPA, and other diseases such as degenerative joint diseases or intervertebral disk displacement. Further studies with a larger sample size are required to confirm the usefulness of CRP measurement for the differential diagnosis of various diseases.

Although little information on the long-term outcomes of IPA dogs is available, Bennett reported that of the 34 IPA dogs in his study, $10(29 \%)$ recovered and 7 showed relapses [4]. In the present study, of the 26 dogs, 10 (38.5\%) had no symptoms even without medication and $16(61.5 \%)$ continued immunosuppressive drug therapy at 6 months after the diagnosis. These results indicate that immunemediated inflammation is transient and immunosuppressive drug therapy can be stopped at least in some IPA dogs. These findings suggest that several etiologies are related to canine IPA; this affects the long-term outcome with regard to medication status.

CRP concentration is known to be elevated in human RA and has been used as an index of disease activity and a prognostic factor $[7,9,19]$. In the present study, the CRP concentrations at the time of diagnosis were markedly elevated in all the IPA dogs, but these could not predict the long-term outcomes of the disease/medication status. However, a significant difference was observed in the CRP concentrations at follow-up (6-13 days after the treatment was started) between the NSM and CM groups, and these concentrations could predict the long-term outcomes in the IPA dogs. Although a correlation was observed between the WBC counts and the CRP concentration at follow-up, the presence 
of leukocytosis did not predict the long-term outcomes of disease/medication status. These results indicated that the initial CRP responses, and not the WBC counts, were useful as an index to predict the outcomes in the IPA dogs. The initial CRP response to corticosteroids and early serial CRP measurements are also known to have a prognostic value in several diseases such as polymyalgia rheumatica [16] and ischemic stroke [20] in human medicine. A potential drawback of our study was that the time point for measuring the CRP concentration varied between 6 and 13 days after the treatment was started. The CRP concentration should preferably be measured under the same conditions (time points or cumulative doses of prednisolone).

In conclusion, based on our preliminary results, we consider that the plasma CRP concentration is a useful laboratory parameter for monitoring the response to therapy in IPA dogs. Furthermore, our study indicated that IPA dogs in whom the CRP concentration normalizes immediately after the initiation of corticosteroid treatment will have a better disease course, and this would be characterized by the discontinuation of the immunosuppressive drugs. Further controlled studies that would evaluate serial CRP concentrations in a larger sample size are needed to confirm this conclusion.

ACKNOWLEDGEMENT. This work was partially supported by the Ministry of Education, Science, Sports and Culture through a Grant-in-Aid for Scientific Research.

\section{REFERENCES}

1. Bennet, D. 1987. Immune-based erosive inflammatory joint disease of the dog: canine rheumatoid arthritis. 1. Clinical, radiological and laboratory investigations. J. Small Anim. Pract. 28: 779-797.

2. Bennet, D. 1987. Immune-based non-erosive inflammatory joint disease of the dog. 1. Canine systemic lupus erythematosus. J. Small Anim. Pract. 28: 871-889.

3. Bennet, D. 1987. Immune-based non-erosive inflammatory joint disease of the dog. 2. Polyarthritis/polymyositis syndrome. J. Small Anim. Pract. 28: 891-908.

4. Bennet, D. 1987. Immune-based non-erosive inflammatory joint disease of the dog. 3. Canine idiopathic polyarthritis. $J$. Small Anim. Pract. 28: 909-928.

5. Ceron, J. J., Eckersall, P. D. and Martynez-Subiela, S. 2005. Acute phase proteins in dogs and cats: current knowledge and future perspectives. Vet. Clin. Pathol. 34: 85-99.

6. Clearfield, M. B. 2005. C-reactive protein: a new risk assessment tool for cardiovascular disease. J. Am. Osteopath. Assoc.
105: 409-416.

7. Dixon, J. S., Bird, H. A., Sitton, N. G., Pickup, M. E. and Wright, V. 1984. C-reactive protein in the serial assessment of disease activity in rheumatoid arthritis. Scand. J. Rheumatol. 13: $39-44$.

8. Dunn, K. J. and Dunn, J. K. 1998. Diagnostic investigations in 101 dogs with pyrexia of unknown origin. J. Small Anim. Pract. 39: 574-580.

9. Gossec, L., Dougados, M., Goupille, P., Cantagrel, A., Sibilia, J., Meyer, O., Sany, J., Daures, J. P. and Combe, B. 2004. Prognostic factors for remission in early rheumatoid arthritis: a multiparameter prospective study. Ann. Rheum. Dis. 63: 675680.

10. Holm, J. L., Rozanski, E. A., Freeman, L. M. and Webster, C. R. L. 2004. C-reactive protein concentrations in canine acute pancreatitis. J. Vet. Emerg. Crit. Care. 14: 183-186.

11. Jacques, D., Cauzinille, L., Bouvy, B. and Dupre, G. 2002. A retrospective study of 40 dogs with polyarthritis. Vet. Surg. 31: 428-434.

12. Jergens, A. E., Schreiner, C. A., Frank, D. E., Niyo, Y., Ahrens, F. E., Eckersall, P. D., Benson, T. J. and Evans, R. 2003. A scoring index for disease activity in canine inflammatory bowel disease. J. Vet. Intern. Med. 17: 291-297.

13. Onishi, T., Inokuma, H., Ohno, K., Soeda, S., Noguchi, K. and Sasaki, K. 2000. C-reactive protein concentrations in normal and diseased dogs-measured by laser nephelometric immunoassay. J. Jpn. Vet. Med. Assoc. 53: 595-601.

14. Patrick, E. H., 2002. Immune-mediated polyarthritis., pp. 1928-1934. In: Textbook of Small Animal Surgery., 3rd ed. (Slatter, D. ed.), W.B.Saunders, Philadelphia.

15. Rondeau, M. P., Walton, R. M., Bissett, S., Drobatz, K. J. and Washabau, R. J. 2005. Suppurative, nonseptic polyarthropathy in dogs. J. Vet. Intern. Med. 19: 654-662.

16. Schreiber, S. and Buyse, M. 1995. The CRP initial response to treatment as prognostic factor in patients with polymyalgia rheumatica. Clin. Rheumatol. 14: 315-318.

17. Shimada, H., Kitabayashi, H., Nabeya, Y., Okazumi, S., Matsubara, H., Funami, Y., Miyazawa, Y., Shiratori, T., Uno, T., Itoh, H. and Ochiai, T. 2003. Treatment response and prognosis of patients after recurrence of esophageal cancer. Surgery. 133: $24-31$.

18. Tecles, F., Spiranelli, E., Bonfanti, U., Ceron, J. J. and Paltrinieri, S. 2005. Preliminary studies of serum acute-phase protein concentrations in hematologic and neoplastic diseases of the dog. J. Vet. Intern. Med. 19: 865-870.

19. van Zeben, D. andBreedveld, F. C. 1996. Prognostic factors in rheumatoid arthritis. J. Rheumatol. Suppl. 44: 31-33.

20. Winbeck, K., Poppert, H., Etgen, T., Conrad, B. and Sander, D. 2002. Prognostic relevance of early serial C-reactive protein measurements after first ischemic stroke. Stroke 33: 24592464. 